\title{
Investigation of Urinary Sestrin2 in Patients with Obstructive Sleep Apnea
}

\author{
Lu Bai ${ }^{1,2} \cdot$ Chunying Sun ${ }^{3} \cdot$ Huifen Zhai ${ }^{4} \cdot$ Chen Chen ${ }^{5} \cdot$ Xiaotian $\mathrm{Hu}^{2} \cdot$ Xiulin $\mathrm{Ye}^{1} \cdot \mathrm{Min} \mathrm{Li}^{1} \cdot$ Yan Fang ${ }^{1,5} \cdot$ Weimin Yang $^{5}$. \\ Haoyan Wang ${ }^{4}$. Shibo Sun ${ }^{1}$ (1)
}

Received: 9 October 2018 / Accepted: 7 February 2019 / Published online: 15 February 2019

(c) The Author(s) 2019

\begin{abstract}
Background Obstructive sleep apnea (OSA) is a disease seriously threatening individual health, which results in serious complications such as hypertension and stroke. These complications are associated with oxidative stress triggered by intermittent hypoxia in OSA. Sestrin2 is a crucial factor involved in oxidative stress. The goal of this study was to investigate if a relationship exists between OSA and Sestrin2.

Methods We prospectively enrolled 71 subjects, and 16 patients of them with severe OSA completed 4 weeks of nasal continuous positive airway pressure (nCPAP) therapy. We measured and compared the concentration of Sestrin2 in the urine of all subjects, as well as the changes between before and after nCPAP treatment. Additionally, the correlation between Sestrin2 and sleep parameters was analyzed, and the multiple linear regression analysis with stepwise selection was performed to explore the relationship between Sestrin2 and various factors.

Results A total of 71 subjects were enrolled and divided into two groups: OSA group $(n=41)$, control group $(n=30)$. The level of urinary Sestrin2 in OSA patients was significantly higher than that of the control group, and increased with the severity of OSA, while it reduced after nCPAP treatment. Additionally, Sestrin 2 was positively correlated with apnea/ hypopnea index (AHI), oxygen desaturation index, oxygen saturation $<90 \%$ percentage of recording time spent (PRTS) and high-density lipoprotein (HDL), while negatively correlated with the lowest oxygen saturation. Importantly, Sestrin2 was independently associated with AHI, oxygen saturation $<90 \%$ PRTS and HDL.
\end{abstract}

Conclusions Urinary Sestrin2 is involved in OSA, and is a paramount marker of OSA severity.

Keywords Sestrin $2 \cdot$ Obstructive sleep apnea $\cdot$ Apnea/hypopnea index $\cdot$ High-density lipoprotein

Shibo Sun

shibosunky@126.com

1 Department of Respiratory and Critical Care Medicine, First Affiliated Hospital, Kunming Medical University, Kunming, China

2015 Innovation Class, Kunming Medical University, Kunming, China

3 Obstetrical Department, Zhengzhou Maternity and Child Care Center, Zhengzhou, China

4 Department of Respiratory Medicine, Beijing Friendship Hospital, Capital Medical University, Beijing, China

5 School of Pharmaceutical Science \& Yunnan Key Laboratory of Pharmacology for Natural Products, Kunming Medical University, Kunming, China

\section{Introduction}

Obstructive sleep apnea (OSA) is a shared sleep-related disorder. According to the survey, the estimated prevalence of OSA is $0.7-3.3 \%$ [1-3]. It is characterized by periodic recurrent episodes of upper airway obstruction during sleep, resulting in apnea [4], and the occurrence of OSA is capable of disrupting sleep integrity, impairing ventilation, and resulting in intermittent hypoxia [5], which seriously lead to coronary artery disease, high blood pressure, cerebrovascular accident, stroke, etc. [6]. There is increasing evidence that these complications are closely related to oxidative stress triggered by intermittent hypoxia [6-9].

Sestrin, originally discovered as p53-inducible proteins, is a highly conserved family of proteins that are thought to protect cells from various damages [10]. The Sestrin family has three distinct members in mammals, which include Sestrin1, 
Sestrin2, and Sestrin3 [11]. Sestrin2, among the three members, also known as hypoxia-inducible gene (Hi95), is an acute response protein that plays a paramount role in various conditions such as excessive oxidative stress, hypoxia, DNA damage, etc. [12]. Moreover, Sestrin2 expression is obviously up-regulated in oxidative stress-related diseases, exerting anti-oxidation, preventing the production of reactive oxygen species (ROS), reducing the accumulation of ROS as an inducible factor of oxidation, and thus protecting cells from oxidative stress $[13,14]$. Chronic intermittent hypoxia (CIH) occurs in patients with OSA during sleeping [15], leading to a strong oxidative stress response [16]. Accordingly, we speculated that Sestrin 2 might be involved in the OSA. To study the relationship between Sestrin2 and OSA may be beneficial in discovering the reasons of oxidative stress-related complications in OSA patients. However, so far, we have not found any relevant reports. The goal of this study was to investigate the changes in urinary Sestrin 2 concentration in OSA patients.

\section{Method}

\section{Research Population}

We prospectively enrolled 71 subjects who were entirely performed by polysomnography (PSG) tests because of snoring, including 41 newly diagnosed OSA patients and 30 healthy controls at the First Affiliated Hospital of Kunming Medical University, from June 2016 to June 2018. The inclusion criteria were patients with 18-65 years old, and the exclusion criteria were patients with heart failure, lung disease, cerebrovascular disease, kidney disease, metabolic diseases like diabetes, and the OSA patients who have been treated before this study.

All patients underwent a systematic physical examination prior to PSG testing to measure the height, weight, waistto-hip ratio, and neck circumference (NC). Their body mass index (BMI) was calculated. The Epworth Sleepiness Score (ESS) was used to assess the degree of daytime sleepiness. This study was approved by the Ethics Committee of the First Affiliated Hospital of Kunming Medical University, and the patient's informed consents were obtained.

\section{PSG}

All subjects were examined by overnight PSG (Alice 5, American) and recorded parameters including EEG, ECG, oral and nasal airflow, oxygen saturation, and chest and abdomen movements. Apnea was defined as a respiratory amplitude reduction $\geq 90 \%$ for more than $10 \mathrm{~s}$. Hypopnea was defined as a decrease in respiratory airflow amplitude reduction $\geq 30 \%$ with a decrease in oxygen saturation $\geq 3 \%$ or arousal [17]. All PSG records were analyzed by two experts to obtain apnea/hypopnea index (AHI). Normal subject was defined with $\mathrm{AHI}<5$, OSA was defined with $\mathrm{AHI} \geq 5$. The hypoxemia index was indicated by oxygen saturation $<90 \%$ percentage of recording time spent (PRTS).

\section{Pressure Titration Under PSG}

An overnight pressure titration was performed under PSG testing using an auto-continuous positive airway pressure (Auto-CPAP) machine (Philips-Respironics REMstarAuto). The airflow signal was collected by the mask pressure signal. The humidifier was routinely used during titration. If the PSG result demonstrated $\mathrm{AHI} \leq 5 / \mathrm{h}, 90$ th percentile recording pressure was defined as the titration pressure.

\section{nCPAP Treatment}

Patients enrolled in the treatment response analysis were treated with a nasal CPAP (nCPAP) machine (Resmed, S9 Escape, Australia). There was a data card on each machine to record the application time. We read the data card and downloaded the data after 4 weeks of treatment. Patients who were treated for more than $4 \mathrm{~h} /$ day were included in the treatment analysis.

\section{Urine Test}

5-7 mL urine specimens were collected from the patients on an empty stomach in the morning after the PSG test and 4 weeks nCPAP treatment, then centrifugated $20 \mathrm{~min}$ by $3000 \mathrm{rpm}$ at $4{ }^{\circ} \mathrm{C}$. Urine supernatant was extracted and stored in $-80^{\circ} \mathrm{C}$ refrigerator for testing. The urinary Sestrin2 concentration was measured with an ELISA kit (Mlbio, Shanghai, China). Other tests including fasting blood glucose, cholesterol, low-density lipoprotein (LDL), high-density lipoprotein (HDL), and urine creatinine were tested by the First Affiliated Hospital of Kunming Medical University. Sestrin2 was adjusted with creatinine: adjust Sestrin $2=($ Sestrin $2 /$ creatinine $) \times 10^{4}$.

\section{Statistical Analysis}

Data were presented as mean \pm standard derivation. In addition, the single-sample Kolmogorov-Smirnov method was used to detect whether the data were normally distributed. Normally distributed data were compared by an unpaired $t$-test, and the data between before and after treatment were compared with paired $t$-test. Rank sum test was used for nonnormally distributed data. Pearson's correlation analysis was applied for correlation analysis. Linear stepwise regression analysis was used to detect the relationship between Sestrin2 and other factors (age, gender, waist-to-hip, BMI, NC, AHI, 
mean oxygen saturation, lowest oxygen saturation, oxygen saturation < 90\% PRTS, ESS, TG, TC, LDL, HDL, fasting blood glucose).

\section{Result}

71 Subjects were prospectively enrolled in this study, and were divided into two groups: OSA group $(39.29 \pm 7.73$ years old, $n=41)$ and control group $(38.70 \pm 8.86$ years old, $n=30$ ). All patient characteristics and the results of urinary Sestrin2 were listed in Table 1. OSA patients were divided into three subgroups according to AHI: mild group $5 \leq \mathrm{AHI}<15$, moderate group $15 \leq \mathrm{AHI}<30$, and severe group AHI $\geq 30$. Sixteen patients with severe OSA completed 4 weeks nCPAP treatment, and the treatment time was greater than $4 \mathrm{~h} /$ day, and then the levels of urinary Sestrin2 after treatment were measured.

The level of urinary Sestrin2 in the OSA group was significantly higher than that of the control group $(P=0.035)$, and as the severity of OSA increased, Sestrin2 level also increased. In addition, Sestrin2 was significantly reduced after nCPAP treatment (Fig. 1). Subgroup analysis demonstrated that the concentration of Sestrin2 in the severe OSA group was significantly higher than that of the other subgroups and control group (Fig. 2).

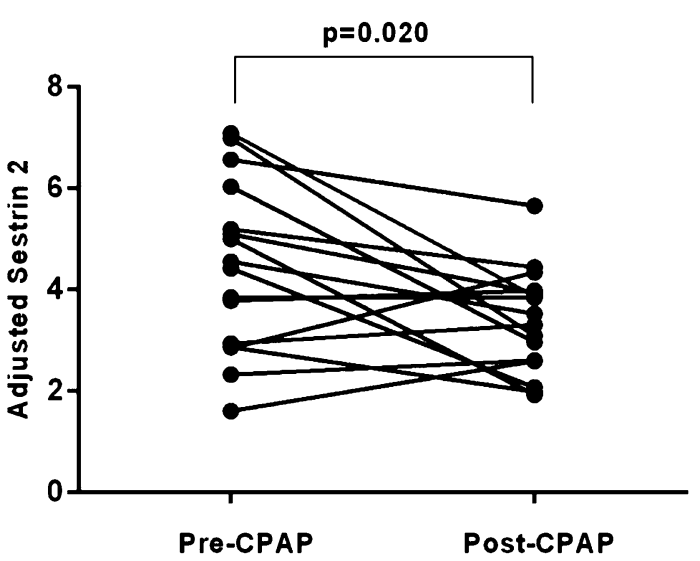

Fig. 1 Results of adjusted Sestrin2 level of 16 severe OSA before and after CPAP treatment
Table 1 Demographics, sleep profiles, blood and urinary measurements of the OSA and control groups

\begin{tabular}{lllc}
\hline & Control group $(n=30)$ & OSA group $(n=41)$ & $P$ values \\
\hline Age $($ years) & $38.70 \pm 8.86$ & $39.29 \pm 7.73$ & 0.765 \\
Gender (male/female) ${ }^{\mathrm{a}}$ & $20 / 10$ & $32 / 9$ & 0.416 \\
BMI $\left(\mathrm{kg} / \mathrm{m}^{2}\right)$ & $27.09 \pm 4.22$ & $28.55 \pm 4.62$ & 0.176 \\
Neck circumference $(\mathrm{cm})$ & $36.06 \pm 5.47$ & $38.56 \pm 6.90$ & 0.093 \\
Waist-to-hip ratio & $0.88 \pm 0.07$ & $0.92 \pm 0.08$ & $0.022^{*}$ \\
AHI (events/h) & $2.32 \pm 1.20$ & $35.00 \pm 20.86$ & $<0.001^{*}$ \\
ODI (events/h) & $1.59 \pm 0.90$ & $31.17 \pm 19.31$ & $<0.001^{*}$ \\
Lowest oxygen saturation (\%) & $90.48 \pm 0.99$ & $79.93 \pm 8.28$ & $<0.001^{*}$ \\
Mean oxygen saturation (\%) & $93.50 \pm 0.94$ & $90.40 \pm 2.91$ & $<0.001^{*}$ \\
Oxygen saturation <90\% PRTS (\%) & $0.46 \pm 1.42$ & $19.35 \pm 8.92$ & $<0.001^{*}$ \\
Total sleep time (min) & $316.8 \pm 38.71$ & $329.83 \pm 40.71$ & 0.179 \\
Arousal index (events/h) & $13.17 \pm 7.32$ & $35.86 \pm 20.63$ & $<0.001^{*}$ \\
Stage of REM $(\%)$ & $13.15 \pm 3.79$ & $11.36 \pm 3.25$ & $0.035^{*}$ \\
Stage of NREM (\%) & $86.85 \pm 3.79$ & $88.64 \pm 3.25$ & $0.035^{*}$ \\
Triglycerides (mmol/L) & $1.51 \pm 0.61$ & $1.77 \pm 1.12$ & 0.224 \\
Sestrin2 (ng/mL creatinine) & $2.69 \pm 1.11$ & $3.39 \pm 1.65$ & $0.035^{*}$ \\
Total cholesterol (mmol/L) & $4.08 \pm 0.80$ & $4.31 \pm 0.64$ & 0.180 \\
Low-density lipoprotein (mmol/L) & $2.31 \pm 0.63$ & $2.51 \pm 0.58$ & 0.160 \\
High-density lipoprotein (mmol/L) & $1.03 \pm 0.29$ & $0.99 \pm 0.24$ & 0.532 \\
Fasting blood glucose (mmol/L) & $4.31 \pm 0.53$ & $4.56 \pm 0.56$ & 0.064 \\
ESS (scores) & $6.73 \pm 2.46$ & $11.92 \pm 4.30$ & $<0.001^{*}$ \\
\hline Values are & &
\end{tabular}

Values are indicated as the mean \pm standard deviation

$B M I$ body mass index, $A H I$ apnea/hypopnea index, $O D I$ oxygen desaturation index, PRTS percentage of recording time spent, REM rapid eye movement, NREM non-rapid eye movement, ESS Epworth Sleepiness Score

${ }^{*} P<0.05$, the results are statistically significant

${ }^{\mathrm{a}}$ Values are indicated as numbers 


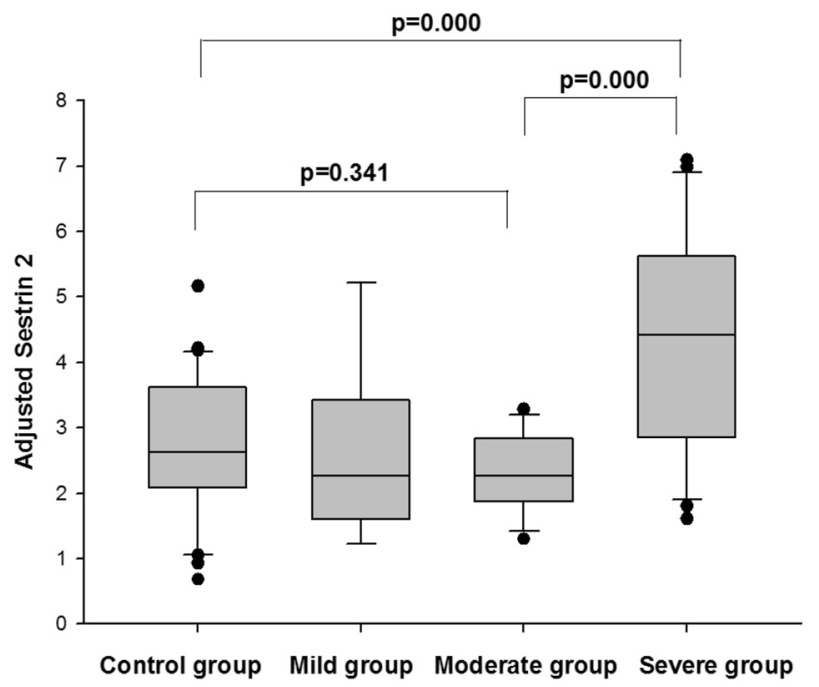

Adjusted Sestrin $2=($ Sestrin $2 /$ Urinary creatinine $) \times 10^{4}$

Fig. 2 Adjusted Sestrin2 levels of control group and OSA subgroups. Adjusted Sestrin $2=($ Sestrin $2 /$ urinary creatinine $) \times 10^{4}$

Sestrin2 was positively correlated with AHI, oxygen desaturation index (ODI), oxygen saturation $<90 \%$ PRTS, HDL, fasting blood glucose, and OSA severity, but negatively correlated with lowest oxygen saturation. Importantly, these relationships still existed after adjusting for age, gender, waist-to-hip, NC, and BMI (Table 2).

The multiple linear regression analysis with stepwise selection showed that Sestrin2 was associated with AHI, oxygen saturation $<90 \%$ PRTS, and HDL (Table 3). However, there was no association between Sestrin2 and waistto-hip ratio.

\section{Discussion}

This study was the first report on investigation of Sestrin 2 in OSA, and the results showed that the Sestrin 2 level elevated in OSA and decreased after nCPAP treatment. In addition, Sestrin2 was positively correlated with AHI, ODI, and oxygen saturation $<90 \%$ PRTS. Most importantly, the multiple regression analysis demonstrated that Sestrin2 was associated with AHI and oxygen saturation $<90 \%$ PRTS. It suggested that Sestrin2 played an important role in OSA.

Several authors $[12,13,18-20]$ suggested that hypoxia, stress, or inflammation led to a growth of directed autophagy of Kelch-like ECH-associated protein 1 (Keap1), thereby decomposed the key regulator of oxidative genes, nuclear erythroid-related factor 2 (Nrf2). The production and accumulation of ROS inhibited by Nrf2 will lead to an increase in Sestrin2 [21, 22]. Moreover, hypoxia, stress, or inflammation will activate the mTORC1. Sestrin2, as a leucine sensor, is
Table 2 Spearman's correlations between adjusted Sestrin2 and the other factors

\begin{tabular}{|c|c|c|c|c|}
\hline & $r$ & $P$ values & $r^{\mathrm{a}}$ & $P$ values \\
\hline Age & 0.170 & 0.156 & & \\
\hline BMI & 0.075 & 0.535 & & \\
\hline Neck circumference & -0.043 & 0.725 & & \\
\hline Waist-to-hip ratio & 0.073 & 0.545 & & \\
\hline AHI & 0.376 & $0.001 *$ & 0.566 & $<0.001^{*}$ \\
\hline ODI & 0.314 & $0.008^{*}$ & 0.559 & $<0.001 *$ \\
\hline Lowest oxygen saturation & -0.358 & $0.002 *$ & -0.350 & $0.004^{*}$ \\
\hline Mean oxygen saturation & -0.160 & 0.183 & -0.138 & 0.268 \\
\hline $\begin{array}{l}\text { Oxygen saturation }<90 \% \\
\text { PRTS }\end{array}$ & 0.378 & $0.001 *$ & 0.562 & $<0.001^{*}$ \\
\hline Total sleep time & -0.047 & 0.698 & 0.044 & 0.726 \\
\hline Arousal index & -0.003 & 0.979 & 0.215 & 0.083 \\
\hline Stage of REM & 0.070 & 0.564 & -0.016 & 0.901 \\
\hline Stage of NREM & -0.070 & 0.564 & 0.016 & 0.901 \\
\hline Triglycerides & -0.054 & 0.654 & -0.030 & 0.814 \\
\hline Total cholesterol & 0.029 & 0.811 & 0.084 & 0.505 \\
\hline Low-density lipoprotein & 0.001 & 0.996 & -0.012 & 0.922 \\
\hline High-density lipoprotein & 0.344 & $0.003 *$ & 0.325 & $0.008 *$ \\
\hline Fasting blood glucose & 0.305 & $0.010^{*}$ & 0.274 & $0.026^{*}$ \\
\hline Epworth Sleepiness Score & 0.298 & $0.011^{*}$ & 0.259 & $0.036^{*}$ \\
\hline Severity of OSA & 0.405 & $0.000 *$ & 0.388 & $0.001 *$ \\
\hline
\end{tabular}

$B M I$ body mass index, $A H I$ apnea/hypopnea index, $O D I$ oxygen desaturation index, PRTS percentage of recording time spent, REM rapid eye movement, NREM non-rapid eye movement, OSA obstructive sleep apnea

$* P<0.05$, the results are statistically significant

${ }^{a}$ Adjusting for age, BMI, gender, waist-to-hip ratio, neck circumference

Table 3 Stepwise multiple regression model of adjusted urinary Sestrin2 levels in the OSA group (adjusted $R^{2}=0.745$ )

\begin{tabular}{lrrr}
\hline & \multicolumn{1}{l}{$B(\mathrm{SE})$} & $\beta$ & $P$ values \\
\hline Constant & $-1.078(0.525)$ & & $0.047^{*}$ \\
AHI & $0.033(0.007)$ & 0.420 & $<0.001^{*}$ \\
Oxygen satura- & $0.094(0.016)$ & 0.509 & $<0.001^{*}$ \\
$\quad$ tion <90\% PRTS & & & \\
HDL & $1.435(0.444)$ & 0.262 & $0.003^{*}$ \\
\hline
\end{tabular}

Independent variables considered: age, gender, BMI, waist-to-hip ratio, neck circumference, AHI, lowest oxygen saturation, oxygen saturation $<90 \%$ PRTS, total sleep time, stage of rapid eye movement sleep, Epworth Sleepiness Score, triglycerides, total cholesterol, lowdensity lipoprotein, HDL, fasting blood glucose

$A H I$ apnea/hypopnea index, $P R T S$ percentage of recording time spent, $H D L$ high-density lipoprotein

$* P<0.05$, the results are statistically significant 
significantly increased in mTORC1 activation [23-25]. It was reported that hypoxia, oxidative stress, and inflammation recurred in OSA [26-28]. In present study, Sestrin2 was positively correlated with ODI and oxygen saturation $<90 \%$ PRTS. In addition, the multiple regression analysis showed that Sestrin2 was associated with oxygen saturation $<90 \%$ PRTS. Therefore, the increasing of urinary Sestrin 2 might be triggered by intermittent hypoxia in OSA.

It was reported that Sestrin2 was a conserved antioxidant protein, and could be activated under pressure to protect cells from oxidative stress [29]. Essler et al. [30] suggested that Sestrin2 upregulation mediated by hypoxia was beneficial to counteracting the production of ROS, and ROS frequently accumulated and increased due to CIH in OSA [4]. Consequently, Sestrin 2 might play an advantageous role in anti-oxidative stress in OSA.

It was reported that nCPAP could significantly reduce ODI and oxygen saturation $<90 \%$ PRTS in patients with OSA, and improve the hypoxia of OSA during sleep [31, 32]. In addition, the nCPAP could improve the oxidative stress and inflammatory response [32, 33]. In this study, the Sestrin 2 reduced with the nCPAP treatment. Hypoxia, which might result in the elevation of Sestrin2, relieved after nCPAP treatment, thus the Sestrin 2 decreased.

It was reported that central adiposity was associated with OSA [34], and it could predict the onset of OSA [35]. In present study, there was no relationship between waist-to-hip ratio and Sestrin2, which meant that the central adiposity had no effect on the change of Sestrin2.

Moreover, sleep architecture impairment of OSA might affect some factors, such as TNF- $\alpha$ [36]. However, in this study, Sestrin2 was not related to REM, NREM sleep, arousal index, and total sleep time, which meant that sleep architecture impairment of OSA did not contribute to increasing Sestrin2 level.

It was reported that there was a significant positive correlation between Sestrin2 and HDL [37]. The HDL played an antioxidant role [38], and the HDL levels were positively correlated with obesity status [39, 40]. Moreover, Sestrin2 regulated the PERK-c/EBP $\beta$ pathway by mediating mTORCI $[41,42]$, and inhibited the ROS-mediated p38 MAPK activation and interfered the expression of uncoupling protein 1 (Ucp1), subsequently resulting in the decrease of heat production and the increase of adipose tissue, which led to obesity $[43,44]$. In addition, HDL was reduced in inflammatory conditions [45], while Sestrin2 could inhibit TLR-mediated inflammatory responses in macrophages [46]. Accordingly, it might be the obesity and inflammation that caused the association between Sestrin 2 and HDL in this study.

However, there was a main limitation that the present study was not randomized; Small sample size was also a limitation. In addition, another limitation was that we used Auto-CPAP titration under PSG for pressure titration instead of the standard CPAP titration, though it was suggested that Auto-CPAP titration under PSG was also effective.

\section{Conclusion}

Urinary Sestrin2 is involved in OSA, and is closely related to the severity of OSA. It decreases with nCPAP treatment. Accordingly, urinary Sestrin 2 is a paramount marker of OSA severity.

\section{Acknowledgements None.}

Funding This study was funded by Yunnan Provincial Department of Education (No. 2017zzx201) and Teaching and Reform Program of Kunming Medical University (Nos. 2016-JY-Y-43, 2017-JY-Y-040).

\section{Compliance with Ethical Standards}

\section{Conflict of interest None.}

Ethical Approval All procedures performed in studies involving human participants were consistent with the Ethical Standards of the Institutional and/or Nation Research Committee, as well as the 1964 Helsinki Declaration and its later amendments or comparable ethical standards.

Informed Consent Informed consent was obtained from all individual participants included in the study.

Open Access This article is distributed under the terms of the Creative Commons Attribution 4.0 International License (http://creativeco mmons.org/licenses/by/4.0/), which permits unrestricted use, distribution, and reproduction in any medium, provided you give appropriate credit to the original author(s) and the source, provide a link to the Creative Commons license, and indicate if changes were made.

\section{References}

1. Lavie P (2002) Incidence of sleep apnea in a presumably healthy working population: a significant relationship with excessive daytime sleepiness. Sleep 25:312-318

2. Gislason T, Almqvist M, Eriksson G et al (1988) Prevalence of sleep apnea syndrome among Swedish men - an epidemiological study. J Clin Epidemiol 41:571-576

3. Bixler EO, Vgontzas AN, Ten Have T et al (1998) Effects of age on sleep apnea in men: I. Prevalence and severity. Am J Respir Crit Care Med 157:144-148. https://doi.org/10.1164/ajrcc m.157.1.9706079

4. Bouloukaki I, Mermigkis C, Kallergis EM et al (2015) Obstructive sleep apnea syndrome and cardiovascular disease: the influence of C-reactive protein. World J Exp Med 5:77-83. https://doi. org/10.5493/wjem.v5.i2.77

5. Elsharkawi I, Gozal D, Macklin EA et al (2017) Urinary biomarkers and obstructive sleep apnea in patients with Down syndrome. Sleep Med 34:84-89. https://doi.org/10.1016/j.sleep.2017.02.005

6. Franklin KA, Lindberg E (2015) Obstructive sleep apnea is a common disorder in the population - a review on the epidemiology of 
sleep apnea. J Thorac Dis 7:1311-1322. https://doi.org/10.3978/j. issn.2072-1439.2015.06.11

7. Flora SJ (2009) Structural, chemical and biological aspects of antioxidants for strategies against metal and metalloid exposure. Oxid Med Cell Longev 2:191-206. https://doi.org/10.4161/ oxim.2.4.9112

8. Dalle-Donne I, Rossi R, Colombo R et al (2006) Biomarkers of oxidative damage in human disease. Clin Chem 52:601-623. https ://doi.org/10.1373/clinchem.2005.061408

9. Finkel T, Holbrook NJ (2000) Oxidants, oxidative stress and the biology of ageing. Nature 408:239-247. https://doi. org/10.1038/35041687

10. Donahoe SM, Stewart GC, McCabe CH et al (2007) Diabetes and mortality following acute coronary syndromes. JAMA 298:765-775. https://doi.org/10.1001/jama.298.7.765

11. Budanov AV, Shoshani T, Faerman A et al (2002) Identification of a novel stress-responsive gene Hi95 involved in regulation of cell viability. Oncogene 21:6017-6031. https://doi.org/10.1038/ sj.onc. 1205877

12. Lee JH, Budanov AV, Karin M (2013) Sestrins orchestrate cellular metabolism to attenuate aging. Cell Metab 18:792-801. https://doi.org/10.1016/j.cmet.2013.08.018

13. Chuang YC, Yang JL, Yang DI et al (2015) Roles of Sestrin2 and ribosomal protein S6 in transient global ischemia-induced hippocampal neuronal injury. Int J Mol Sci 16:26406-26416. https://doi.org/10.3390/ijms161125963

14. Rai N, Kumar R, Desai GR et al (2016) Relative alterations in blood-based levels of Sestrin in Alzheimer's disease and mild cognitive impairment patients. J Alzheimers Dis 54:1147-1155. https://doi.org/10.3233/JAD-160479

15. Zhou L, Chen P, Peng Y et al (2016) Role of oxidative stress in the neurocognitive dysfunction of obstructive sleep apnea syndrome. Oxid Med Cell Longev 2016:9626831

16. Eisele HJ, Markart P, Schulz R (2015) Obstructive sleep apnea, oxidative stress, and cardiovascular disease: evidence from human studies. Oxid Med Cell Longev 2015:608438. https:// doi.org/10.1155/2015/608438

17. Dumitrascu R, Heitmann J, Seeger W et al (2013) Obstructive sleep apnea, oxidative stress and cardiovascular disease: lessons from animal studies. Oxid Med Cell Longev 2013:234631. https ://doi.org/10.1155/2013/234631

18. The Report of an American Academy of Sleep Medicine Task Force (1999) Sleep-related breathing disorders in adults: recommendations for syndrome definition and measurement techniques in clinical research. Sleep 22:667-689

19. Li K, Chen Z, Qin Y et al (2018) MiR-664a-3p expression in patients with obstructive sleep apnea: a potential marker of atherosclerosis. Medicine (Baltim) 97:e9813. https://doi. org/10.1097/MD.0000000000009813

20. Ye J, Wang M, Xu Y et al (2017) Sestrins increase in patients with coronary artery disease and associate with the severity of coronary stenosis. Clin Chim Acta 472:51-57. https://doi. org/10.1016/j.cca.2017.07.020

21. Fu H, Song W, Wang Y et al (2018) Radiosensitizing effects of Sestrin2 in PC3 prostate cancer cells. Iran J Basic Med Sci 21:621-624. https://doi.org/10.22038/IJBMS.2018.18283.4923

22. Bae SH, Sung SH, Oh SY et al (2013) Sestrins activate Nrf2 by promoting p62-dependent autophagic degradation of Keap1 and prevent oxidative liver damage. Cell Metab 17:73-84. https:// doi.org/10.1016/j.cmet.2012.12.002

23. Zeng N, D'Souza RF, Sorrenson B et al (2018) The putative leucine sensor Sestrin2 is hyperphosphorylated by acute resistance exercise but not protein ingestion in human skeletal muscle. Eur J Appl Physiol 118:1241-1253. https://doi.org/10.1007/s0042 $1-018-3853-8$
24. Lee M, Kim JH, Yoon I et al (2018) Coordination of the leucine-sensing Rag GTPase cycle by leucyl-tRNA synthetase in the mTORC1 signaling pathway. Proc Natl Acad Sci USA 115:E5279-E5288. https://doi.org/10.1073/pnas.1801287115

25. Wolfson RL, Sabatini DM (2017) The dawn of the age of amino acid sensors for the mTORC1 pathway. Cell Metab 26:301-309. https://doi.org/10.1016/j.cmet.2017.07.001

26. Tóthová L, Hodosy J, Mucska I et al (2014) Salivary markers of oxidative stress in patients with obstructive sleep apnea treated with continuous positive airway pressure. Sleep Breath 18:563-570. https://doi.org/10.1007/s11325-013-0919-z

27. Malhotra A, White DP (2002) Obstructive sleep apnoea. Lancet 360:237-245. https://doi.org/10.1016/S0140-6736(02)09464-3

28. Edwards KM, Tomfohr LM, Mills PJ et al (2011) Macrophage migratory inhibitory factor (MIF) may be a key factor in inflammation in obstructive sleep apnea. Sleep 34:161-163

29. Zhang LL, Zhang ZJ (2018) Sestrin2 aggravates oxidative stress of neurons by decreasing the expression of Nrf2. Eur Rev Med Pharmacol Sci 22:3493-3501. https://doi.org/10.26355/eurrev_20180 6_15176

30. Essler S, Dehne N, Brune B (2009) Role of Sestrin2 in peroxide signaling in macrophages. FEBS Lett 583:3531-3535. https://doi. org/10.1016/j.febslet.2009.10.017

31. Zhang J, Tan H, Shi XN et al (2006) The effect of nasal continuous positive airway pressure on endothelial function in obstructive sleep apnea-hypopnea syndrome with coronary heart disease. Zhonghua Nei Ke Za Zhi 45:188-191

32. Christou K, Kostikas K, Pastaka C et al (2009) Nasal continuous positive airway pressure treatment reduces systemic oxidative stress in patients with severe obstructive sleep apnea syndrome. Sleep Med 10:87-94. https://doi.org/10.1016/j.sleep.2007.10.011

33. Schulz R, Mahmoudi S, Hattar K et al (2000) Enhanced release of superoxide from polymorphonuclear neutrophils in obstructive sleep apnea. Impact of continuous positive airway pressure therapy. Am J Respir Crit Care Med 162:566-570. https://doi. org/10.1164/ajrccm.162.2.9908091

34. Schäfer H, Pauleit D, Sudhop T et al (2002) Body fat distribution, serum leptin, and cardiovascular risk factors in men with obstructive sleep apnea. Chest 122:829-839

35. Gaines J, Vgontzas AN, Fernandez-Mendoza J et al (2016) Inflammation mediates the association between visceral adiposity and obstructive sleep apnea in adolescents. Am J Physiol Endocrinol Metab 311:E851-E858. https://doi.org/10.1152/ajpendo.00249 .2016

36. Hirotsu C, Albuquerque RG, Nogueira $\mathrm{H}$ et al (2017) The relationship between sleep apnea, metabolic dysfunction and inflammation: the gender influence. Brain Behav Immun 59:211-218. https ://doi.org/10.1016/j.bbi.2016.09.005

37. Nourbakhsh M, Sharifi R, Ghorbanhosseini SS (2017) Evaluation of plasma TRB3 and Sestrin-2 levels in obese and normalweight children. Child Obes 13:409-414. https://doi.org/10.1089/ chi.2017.0082

38. Kotani K, Tsuzaki K, Taniguchi N et al (2013) Correlation between reactive oxygen metabolites and atherosclerotic risk factors in patients with type diabetes mellitus. Indian J Med Res 137:742-748

39. Nigro E, Scudiero O, Monaco ML et al (2014) New insight into adiponectin role in obesity and obesity-related diseases. Biomed Res Int 2014:658913. https://doi.org/10.1155/2014/658913

40. Christou GA, Kiortsis DN (2013) Adiponectin and lipoprotein metabolism. Obes Rev 14:939-949. https://doi.org/10.1111/ obr.12064

41. Soontornniyomkij V, Soontornniyomkij B, Moore DJ et al (2012) Antioxidant sestrin-2 redistribution to neuronal soma in human immunodeficiency virus-associated neurocognitive disorders. J 
Neuroimmune Pharmacol 7:579-590. https://doi.org/10.1007/ s11481-012-9357-0

42. Park HW, Park H, Ro SH et al (2014) Hepatoprotective role of Sestrin2 against chronic ER stress. Nat Commun 5:4233. https:// doi.org/10.1038/ncomms5233

43. Kotani K, Fujiwara S, Tsuzaki K et al (2011) The association between the uncoupling protein-1 gene A-3826G polymorphism and high-density lipoprotein cholesterol in a general japanese population: a consideration of the obesity status. J Clin Med Res 3:319-324. https://doi.org/10.4021/jocmr738w

44. Ro SH, Nam M, Jang I et al (2014) Sestrin2 inhibits uncoupling protein 1 expression through suppressing reactive oxygen species. Proc Natl Acad Sci USA 111:7849-7854. https://doi.org/10.1073/ pnas. 1401787111
45. Barter P (2002) Effects of inflammation on high-density lipoproteins. Arterioscler Thromb Vasc Biol 22:1062-1063

46. Yang JH, Kim KM, Kim MG et al (2015) Role of sestrin2 in the regulation of proinflammatory signaling in macrophages. Free Radic Biol Med 78:156-167. https://doi.org/10.1016/j.freeradbio med.2014.11.002

Publisher's Note Springer Nature remains neutral with regard to jurisdictional claims in published maps and institutional affiliations. 